\title{
Re: Randomized Controlled Trial to Compare the Safety and Efficacy of Tamsulosin, Solifenacin, and Combination of Both in Treatment of Double-J Stent-Related Lower Urinary Symptoms
}

Yousef Rezaei

Students' Research Committee, Urmia University of Medical Sciences, Urmia, Iran

Vol. 39 (6): 832-840, November - December, 2013

To the editor,

I read the newly published article entitled "Randomized Controlled Trial to Compare the Safety and Efficacy of Tamsulosin, Solifenacin, and Combination of Both in Treatment of Double-J Stent-Related Lower Urinary Symptoms" by Shalaby and associates (1). They have concluded that combined tamsulosin and solifenacin regimen is effective to alleviate double-J stent related symptoms in comparison with both monotherapies. Ureteral stents, especially double-J stents, are associated with some discomforts and strategies implemented to attenuate those, including exact stent indications, stent insertion maneuvers, designation and administering drugs have been proposed so far (2). Given these notions, only three articles, including the mentioned study, have shown the effect of combination of adrenergic alpha-1 antagonist and anti-cholinergic on relieving ureteral stent-related symptoms $(3,4)$. All investigations have had some limitations based on study methods, but their findings are of great value for designing complementary investigations. Thanks to the investigators for their well-designed trial, but there are some thoughts to be mentioned.

The first concern is that all procedures including ureterorenoscopy (URS)/ureterolithotomy, percutaneous nephrolithotomy (PCNL), extracorporeal shock wave lithotripsy (ESWL) and endopyelotomy were enrolled in that study, while it could influence the results especially in the third and fourth groups of study because of including more composite of PCNL, ESWL and endopyelotomy cases compared with control group (26.25\% versus 31\% and 25\% versus 31\%, respectively). All types of surgeries might have different complications and consequently varied symptoms interfered with the effect of studied drugs. ESWL compared with ureteroscopy has lower complications consisted of flank pain and dysuria; however, stone-free rate has been shown to be more in those undergoing ureteroscopic procedures $(5,6)$. Moreover, PCNL in comparison with ESWL presents more stone-free status, although it is accompanied by more flank pain and major postoperative complications (7). Endopyelotomy is also associated with less pre- and post-surgical complications (8). Theses confounding factors might interfere with their conclusion; hence we cannot exactly state that their findings are attributable to either the drugs' effect or having more number of surgeries with low complications allocated in some groups. Regarding such confounding factors, it is reasonable to conduct separate analyses only for those undergone URS as a main intervention in all groups, about 70\% in all groups. Since providing further analyses will be helpful to differentiate between drug- and procedure-related impacts on evaluated scores, and to make the 
analyzed cohort more homogeneous. Recently published randomized trial with main aim similar to this study (4), has demonstrated findings close to this trial, although its contributors have analyzed the type of surgeries (URS and PCNL) separately and have shown findings, which were more generalizable.

Second concern is about the presentations of analyses through the article. As the authors reported in result section, pre- and post-insertion assessed scores showed some significant differences; however, these data were not reported in tables and only pre-insertion and 2 weeks post-insertion have been tabulated. In addition, if authors used on-way repeated measures ANOVA, reporting the p-value of this analysis is mandatory along with one-way ANOVA test. And also, there are no mention about analyses between control group and each monotherapy groups, which can be more useful than comparing monotherapy groups as shown in Table-3. Post-hoc test used for paired analysis has not also been reported for pre-insertion scores, which are of great importance to show the lack of significant difference regarding baseline scores between groups.

Finally, the combined regimen of alpha-adrenergic and anti-cholinergic is a new effective strategy in urology settings for alleviating lower urinary tract symptoms in the background of some diseases, especially following urinary tract manipulation. Further large-scale clinical trials are needed to confirm this regimen for using in practice.

\section{REFERENCES}

1. Shalaby E, Ahmed AF, Maarouf A, Yahia I, Ali M, Ghobish A: Randomized controlled trial to compare the safety and efficacy of tamsulosin, solifenacin, and combination of both in treatment of double-j stent-related lower urinary symptoms. Adv Urol. 2013;2013:752382.

2. Haleblian G, Kijvikai K, de la Rosette J, Preminger G: Ureteral stenting and urinary stone management: a systematic review. J Urol. 2008;179:424-30.

3. Lim KT, Kim YT, Lee TY, Park SY: Effects of tamsulosin, solifenacin, and combination therapy for the treatment of ureteral stent related discomforts. Korean $\mathrm{J}$ Urol. 2011;52:485-8.

4. Tehranchi A, Rezaei $Y$, Khalkhali $H$, Rezaei M: Effects of terazosin and tolterodine on ureteral stent related symptoms: a double-blind placebo-controlled randomized clinical trial. Int Braz J Urol. 2013;39:832-40.

5. Aboumarzouk OM, Kata SG, Keeley FX, McClinton S, Nabi G: Extracorporeal shock wave lithotripsy (ESWL) versus ureteroscopic management for ureteric calculi. Cochrane Database Syst Rev. 2012;5:CD006029.
6. Pearle MS, Nadler R, Bercowsky E, Chen C, Dunn M, Figenshau RS, et al.: Prospective randomized trial comparing shock wave lithotripsy and ureteroscopy for management of distal ureteral calculi. J Urol. 2001;166:1255-60.

7. Huang Z, Zhao X, Zhang L, Zhong Z, Xu R, Zhang L: Extracorporeal shock wave lithotripsy for management of residual stones after ureterolithotripsy versus minipercutaneous nephrolithotomy: a retrospective study. PLoS One. 2013;8:e67046.

8. Dobry E, Usai P, Studer UE, Danuser H: Is antegrade endopyelotomy really less invasive than open pyeloplasty? Urol Int. 2007;79:152-6.

\footnotetext{
Correspondence address: Yousef Rezaei, MD Students' Research Committee, Urmia University of Medical Sciences, Urmia, Iran Fax: + 98 441-293-0800 E-mail: yousefrezaei1986@gmail.com
} 